\title{
Currents, Stress Tensor and Generalized Unitarity in Conformal Invariant Quantum Field Theory
}

\author{
G. MACK \\ Institut für Theoretische Physik der Universitat Bern, Bern \\ K. SYMANZIK \\ Deutsches Elektronen-Synchrotron DESY, Hamburg
}

Received May 3, 1972

\begin{abstract}
Matrix elements of internal symmetry currents and energy momentum density tensor are constructed in Migdal Polyakov conformal invariant bootstrap field theory. Their 3-point functions satisfy Bethe Salpeter equations which determine any free coefficients that may still occur in the conformal invariant Ansatz. Ward identities are verified for all $n$-point functions. They imply correct equal time current commutation relations. A proof of generalized unitarity is also given. Various equivalent forms of the propagator bootstrap are discussed. Our algebraic techniques also yield an eigenvalue equation for first order correction to the exactly conformal invariant theory, assuming the latter is Gell-Mann Low large momentum asymptote of a renormalizable finite mass theory.
\end{abstract}

\section{Introduction}

The Migdal-Polyakov bootstrap approach [1-5] to construct a conformal invariant quantum field theory offers an interesting alternative to the up to now only available canonical perturbation theory. This new approach has been shown [5] to be free from UV and infrared divergences. Moreover, it may be hoped to reproduce correctly the behavior of realistic strong interaction quantum field theory (QFT) in a selected class of high energy limits, since it appears [6] that the Gell-Mann Low limit $[7,8]$ of perturbation theoretically renormalizable theories indeed is conformal invariant ${ }^{1}$.

For the physical interpretation of the theory one should show how to extract observables. Since one is dealing with an infraparticle theory

1 The crucial point is the softness for the trace of the stress tensor in the finite mass theory, which was proven recently by Schroer [6]. Use of conformal symmetry was advocated long ago by Wess and Kastrup [9]. That the divergence of the dilation current should be a soft operator, i.e. emphasize low frequencies, was first conjectured in Ref. [10]. The discussion was extended to conformal symmetry in Ref. [11]. The hypothesis became powerful after Wilson combined it with the notion of operator product expansion [12].

17 Commun math. Phys., Vol 27 
[13] an $S$-matrix does not exist [12]. Therefore, and also as a further check of the consistency of the theory, one looks for other observables characteristic for local QFT: local charge and current, and local energy momentum, i.e. a stress tensor. These should satisfy the appropriate Bethe-Salpeter equations to guarantee that they are local operators, and Ward-Takahashi identities which identify them.

Furthermore, if the theory is a local QFT it must also satisfy the axiomatic positivity requirements, for which generalized unitarity [14] is sufficient. One knows that a massive theory does satisfy generalized unitarity if its Green functions have the many-particle structure [15] familiar from canonical perturbation theory. For the many-point Green functions, these structure properties are manifest in skeleton expansions. For the vertex and the propagator, they are assured by their integral equations (here called bootstraps). Generalized unitarity will then also hold for the Gell-Mann Low limit theory if the massive theory considered is a renormalizable Lagrangian one.

However, it seems nevertheless desirable to attempt a direct verification of generalized unitarity for the conformal invariant Migdal-Polyakov theory since one does not want to start with the assumption that it is Gell-Mann Low limit of a massive theory.

These are the problems to be studied in the present paper. In going along, we shall also have occasion to study integral equations for the 3 -point functions and, especially, the 2-point functions [16]. They provide convenient alternative forms of the propagator bootstrap, more tractable than unitarity type relations because analytic continuation to euclidean space [17] can be performed. This avoids the difficulties inherent in the use of conformal invariance in Minkowski space [18].

Conformal invariant QFT is a noncanonical theory. It relies on Wilson's idea [12] of dynamical dimensionality of fields, which in turn signals the breakdown of canonical equal-time commutation relations. Therefore, one cannot use the canonical formalism to infer existence of conserved local currents associated with symmetries. An explicit demonstration is needed ${ }^{2}$.

We will explain in detail how to construct Green functions involving internal symmetry currents (Section 2) or the stress energy tensor (Section 3). It will be proven in Section 4 that all the Green functions so constructed satisfy the appropriate Ward Takahashi identities, as a consequence of the bootstrap equations stated in Section 1. We remark that Ward identities are not true skeleton graph by skeleton graph, as

${ }^{2}$ A demonstration of gauge invariance in renormalized perturbation theory which does not use the canonical formalism (i.e. a regularized Lagrangian) has been given by Brandt [19] for Q.E.D. Some of our algebra has been inspired by his. 
is known from canonical perturbation theory ${ }^{3}$. It will be shown that they are restored after summing up. The Ward identities are also obeyed by Green functions involving more than one current. This assures that the currents satisfy correct equal-time commutation relations.

In conformal invariant bootstrap field theory, locality and spectrum condition are satisfied order by order in the skeleton graph expansion. This result is implicit in Ref. [20]. It will be shown in Section 5 that also generalized (off mass shell) unitarity relations are satisfied ${ }^{4}$ (after summing up) but only for the presumably discrete set of values of coupling constant and dynamical dimensions of fields which satisfy the bootstrap conditions. This is to be contrasted with canonical perturbation theory, where locality and spectrum conditions are also true order by order, while unitarity is fulfilled qua relation between formal power series, i.e. in a sense identically in the coupling constant [21].

The graphical notation used throughout the paper is explained in Appendix A. The Appendix B contains a short remark on the smalldistance behavior of some functions involved in the Ward identities. Finally, in Appendix $\mathrm{C}$ we review some implications of the results of Ref. [8] for the expected connection with massive theory and give an example of how to compute corrections to the exactly conformal invariant theory.

Not considered in this paper is the problem of extracting from the conformal invariant theory predictions for observables of the realistic finite mass theory. Apart from the total $e^{+} e^{-}$-annihilation cross section into hadrons (see Section 2) this always requires consideration of Green functions at exceptional momenta, where the asymptotics is complicated by infrared effects, as was discussed in Ref. [8].

\section{Integral Equations}

Construction of a conformal invariant field theory is based on the observation of Migdal [1,2] and Polyakov [3] that integral equations for dressed vertex (and propagator [4]) allow for a conformal invariant solution.

The integral equation for the vertex reads

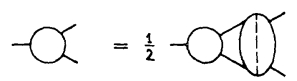

3 If in perturbation theory one inserts the complete expansion for vertex and propagator into a truncated skeleton graph expansion, some graphs of sufficiently high order in the coupling constant are included while others of the same order are not included. As a consequence such an approximation is not gauge invariant.

${ }^{4}$ Strictly speaking, certain convergence assumptions are made in order to guarantee uniqueness of the solution of Eq. (5.4c). For these to hold, negligibility of infinitely often reducible graphs is sufficient but not necessary. (Cp. also Appendix B.) 
where the right hand side (RHS) involves the Bethe Salpeter (BS) kernel. An inhomogeneous term (bare vertex) is absent because it would violate conformal symmetry. This is in agreement with the compositiness condition $Z_{1}=Z_{3}=0$. Eq. (1.1) will be referred to as the "vertex bootstrap".

As an integral equation for the dressed propagator one can use the off-mass-shell unitarity relation for the 2-point function in the form proposed by Polyakov [22]. From a computational point of view it has turned out to be more convenient to use a renormalized form of the Schwinger Dyson equation for the 2-point function. Such an equation was derived by one of us some time ago [16] for $\phi^{3}$ theory in six dimensions. Analogous equations for $\phi^{4}$ theory in four dimensions were given by $\mathrm{Wu}$ [23], Taylor [24], and Johnson [25]. These equations are in essence a rewriting of the presciptions of the Bogoliubov-ParasiukHepp renormalization technique, and we will discuss them at the end of Section 5. For the purpose of this paper other, with respect to the resulting bootstrap equivalent, equations are more interesting. They will now be described:

For general $n$-point vertex functions (including for $n=2$ the negative inverse propagator), Bethe Salpeter kernel etc. let us define

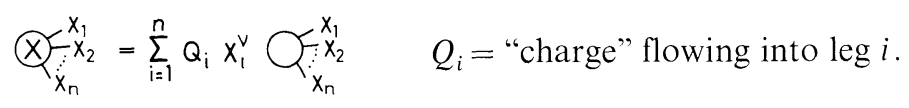

In particular, for the dressed 3-point vertex and the dressed propagator

$$
\begin{aligned}
-\bigotimes & =D_{v}-\bigcirc=\sum_{i}^{3} Q_{i} x_{i}-\bigcirc \\
* & =D_{l}-=Q\left(x_{i}^{v}-x_{f}^{v}\right) \overline{x_{1} x_{f}} ; \quad Q=\text { "charge" flowing from } i \text { to } f .
\end{aligned}
$$

$Q$ may be either a conserved internal charge, or a component $P^{\mu}$ of momentum, with $\mu \neq v$ so that $\left[P^{\mu}, x^{\nu}\right]_{-}=0$.

With this notation, the integral equation for the dressed negative inverse propagator $\left(=2\right.$-point vertex function) reads ${ }^{5}$

$$
-\frac{x^{-1}}{x_{1}}=-\mathrm{O}^{*} \mathrm{O}-+\frac{1}{4}-\mathrm{Ox} \mathrm{O}
$$

It involves again the Bethe Salpeter kernel. The equation is finite and well defined by the criteria of Ref. [5] for noncoinciding external coordinates $x_{l} \neq x_{f}$. Extension to a distribution defined for all $x_{i}, x_{f}$ is trivial and unique because of dilatation invariance.

\footnotetext{
${ }^{5}$ In all of the following, we insert combinational factors as appropriate for one hermitean field, such that an internal charge $Q$ is represented by a matrix and the familiar sign rule for loops from Fermi components is to be observed.
} 
We remark that Eq. (1.2b) is meaningful and valid also in renormalized perturbation theory, for $x_{i} \neq x_{f}$. The proof of this fact parallels that given in Ref. [16] for Eq. (5.8) below.

If a propagator carries some internal charge, one may use either the "charge form" or the "momentum form" of $(1.2 b)$. They are obtained by choosing $Q=$ internal charge, or $Q=$ momentum $P^{\mu}$ in definition (1.2a). Only one of them is needed as a dynamical equation, but both forms give equivalent results. In fact there is still another equivalent form of the propagator bootstrap (1.2b), for each choice of $Q$. It is obtained from the B.S. equation for a conserved current resp. stress tensor's 3-point function and is given in Eqs. (2.11) resp. (3.19) below. Use of Eq. (2.11) as a bootstrap equation for charged propagators was independently proposed by Migdal ${ }^{6}$. We shall show in Section 4 and 5 that all these various forms of the propagator bootstrap are equivalent, modulo validity of the vertex bootstrap (1.1), to imposing the generalized unitarity condition for the 2-point function, Eq. (5.5) below. They are then a fortiori equivalent to each other.

Eqs. (1.1) and (1.2) are solved by the unique conformal invariant Ansatz for dressed propagator and 3-point vertex Eqs. (1.5), (1.7) below, and one obtains a set of algebraic equations to determine coupling constants and dynamical dimensions of the fields.

The higher $n$-point vertex functions, $n \geqq 4$, and the Bethe Salpeter kernel are to be constructed by skeleton graph expansion in terms of dressed propagator and 3-point vertices. In particular, the Bethe Salpeter kernel is:

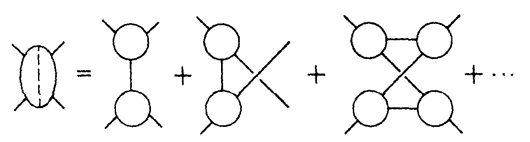

The factors $Q x^{v}$ resp. $P^{\mu} x^{v}(v \neq \mu)$ multiplying the BS kernel in (1.2b) may be distributed within any BS graph in an obvious way:

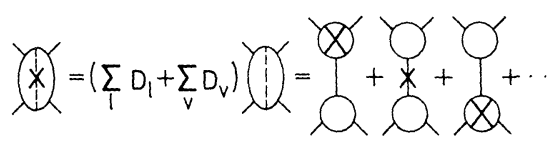

Summation is over all possibilities of attaching a cross to a line or a vertex within any skeleton graph. All contributions proportional $Q x_{a}$, with $x_{a}$ an integration variable, cancel out because of charge resp. momentum conservation.

Throughout the present paper we shall adopt normalization of the propagator as $G(x, 0)=2^{d} \Gamma(d)\left(-x^{2}+i \varepsilon\right)^{-d}$ resp. $i \nabla_{x} 2^{d-\frac{1}{2}} \Gamma\left(d-\frac{1}{2}\right)\left(-x^{2}+i \varepsilon\right)^{\frac{1}{2}-d}$ where $d$ is the dynamical dimension of mass of the field involved.

${ }^{6}$ Migdal, private communication through Todorov. 
Full conformal invariance implies a selection rule

$$
\langle 0|\phi(x) \psi(y)| 0\rangle=0 \text { if } \quad d_{\phi} \neq d_{\psi} .
$$

Because of the explicit factors $x$ involved in (1.2b) (which spoil manifest conformal invariance) it is not evident that selection rule(1.6) is reproduced by Eq. (1.2b). However, in the commonly discussed models there is only at most one fundamental field for any given spin and internal quantum numbers. Selection rule (1.6) is then automatic. On the other hand, Eq. (1.2b) is manifestly dilatation symmetric, and with selection rule (1.6) assured, conformal invariance of the propagator follows already from dilatation symmetry and $\gamma_{5}$-invariance.

For later convenience, we will also reproduce the unique conformal invariant expression for the 3-point vertex function

$$
\Gamma\left(x_{1} x_{2} x_{3}\right)=-i g \Gamma\left(D-\Sigma \delta_{i}\right)^{-1} x_{12}^{-2 \delta_{3}} x_{13}^{-2 \delta_{2}} x_{23}^{-2 \delta_{1}}
$$

where $x_{i j}^{\alpha}=\left(-\left(x_{i}-x_{j}\right)^{2}+i \varepsilon\right)^{\alpha / 2}, D=N o$. of space time dimensions and $\delta_{1}=\delta_{2}=\delta_{3}=\frac{1}{2}(D-d)$ if all fields have the same dynamical dimension $d$.

\section{Currents Associated with Internal Symmetries}

Let us consider a theory which is symmetric under some compact semisimple $m$-parameter internal-symmetry group $G$ - for instance chiral $S U(3) \times S U$ (3). That means the Green functions are unchanged under an infinitesimal transformation of fields

$$
\delta \underline{\phi}(x)=\varepsilon_{a} X^{a} \underline{\phi}(x)
$$

with matrices $X^{a}$ forming a (in general reducible) skew hermitean representation of the Lie algebra of $G$. $\phi$ denotes a column vector composed of all the basic fields of the theory.

In such a theory there ought to exist local currents $j_{\mu}^{a}(x), a=1 \ldots m$, such that

$$
\left[\underline{\phi}(x), j_{0}^{a}(0)\right]_{x^{0}=0}=-i X^{a} \underline{\phi}(x) \delta(\mathbf{x})+\text { S.T., } \quad a=1 \ldots m ;
$$

S.T. stands for gradient terms.

As is well known ${ }^{7}$, commutation relations (2.2) translate into Ward Takahashi identities for the time ordered Green functions of the theory. They may be stated most compactly in the language of generating functionals. Let

$$
\begin{gathered}
G_{\mu}^{a}\left(x ; y_{1} \ldots y_{n}\right)=\left\langle 0\left|T^{*}\left\{j_{\mu}^{a}(x) \underline{\phi}\left(y_{1}\right) \ldots \underline{\phi}\left(y_{n}\right)\right\}\right| 0\right\rangle_{\mathrm{conn} .}, \\
G\left(y_{1} \ldots y_{n}\right)=\left\langle 0\left|T\left\{\underline{\phi}\left(y_{1}\right) \ldots \underline{\phi}\left(y_{n}\right)\right\}\right| 0\right\rangle_{\mathrm{conn} .}
\end{gathered}
$$

${ }^{7}$ The effect of Schwinger terms in Eq. (2.2) may be compensated by appropriate choice of seagulls such that one may demand that "normal" Ward identities (2.5) hold. See Ref. [26]. 
the connected Green functions, and $J(y)$ a row vector of (possibly anticommuting, $c$-number) source functions such that the scalar product $\underline{J}(y) \phi(y)$ may be formed. The generating functionals $G_{\mu}^{a}(x, J\}$ and $G\{J\}$ are then constructed in the usual way, viz. (cp. e.g., Sections II.2-3 of Ref. [27])

$$
G_{\mu}^{a}(x, \underline{J}\}=\sum_{k !} \frac{i^{k}}{k !} \int d y_{1} \ldots d y_{k} \underline{J}\left(y_{1}\right) \ldots \underline{J}\left(y_{k}\right) G_{\mu}^{a}\left(x ; y_{1} \ldots y_{k}\right)
$$

etc. The Ward Takahashi identities (WTI) are

$$
\nabla_{\lambda}^{\mu} G_{\mu}^{a}(x, \underline{J}\}=i \underline{J}(x) X^{a} \underline{G}_{x}\{\underline{J}\} \text {, where } \underline{G}_{x}\{J\} \equiv \frac{\delta}{\delta \underline{J}(x)} G\{\underline{J}\} .
$$

It suffices to consider the currents associated with one generator (charge) out of every simple component of the symmetry Lie algebra. The remaining currents may then be obtained by applying an internal symmetry transformation. We may therefore without loss of generality take matrix $X^{a}$ to be diagonal (the label $a$ will be dropped henceforth):

$$
X_{\alpha \beta}=-i Q_{x} \delta_{\alpha \beta} \quad \text { (no summation). }
$$

For the nonvanishing 3-point Green functions one obtains then from WTI (2.5)

$$
\nabla_{x}^{\mu} G_{\mu}(x ; y, z)=Q G(y, z)\{\delta(y-x)-\delta(z-x)\}
$$

where $Q$ is the charge of the component field $\phi(y)$, and $G(.,$.$) its 2-point$ function.

It will be helpful to write down also the differentiated Ward identity for the proper 3-point vertex function $\Gamma_{\mu}(x ; y, x)$. This proper vertex is obtained from the Green function $G_{\mu}(x ; y, z)$ by amputation on arguments $y$ and $z$, viz.

$$
G_{\mu}(x ; y, z)=\iint d y^{\prime} d z^{\prime} G\left(y, y^{\prime}\right) \Gamma_{\mu}\left(x ; y^{\prime}, z^{\prime}\right) G\left(z, z^{\prime}\right) .
$$

As a consequence of WTI (2.6) its Fouriertransform

$$
\tilde{\Gamma}_{\mu}\left(p_{1} ; p_{2}, p_{3}\right)(2 \pi)^{D} \delta\left(\Sigma p_{i}\right) \equiv \int d x_{1} \ldots d x_{3} e^{\Sigma p^{2 x}} \Gamma_{\mu}\left(x_{1} ; x_{2}, x_{3}\right)
$$

satisfies the Ward identity

$$
\check{\Gamma}^{\mu}(0 ; p,-p)=Q \nabla_{p}^{\mu} \tilde{G}^{-1}(p,-p) .
$$

In configuration space, momentum differentiation $i \nabla_{p}^{\mu}=x_{f}^{\mu}-x_{i}^{\mu}$.

The $n$-point vertex function $\Gamma_{\mu}\left(x ; y_{1} \ldots y_{n}\right)$ is the one particle irreducible part of the connected Green function $G_{\mu}\left(x ; y_{1} \ldots y_{n}\right)$, amputated with respect to arguments $y_{1} \ldots y_{n}$. It satisfies WTI which are obtained from (2.5) most conveniently by a functional Legendre transform, as explained 
in detail in Ref. [27]. In written-out form they read

$$
\nabla_{\lambda}^{\mu} \Gamma_{\mu}\left(x ; y_{1} \ldots y_{n}\right)=\sum_{i} Q_{i} \delta\left(x-y_{i}\right) \Gamma\left(y_{1} \ldots y_{n}\right)
$$

where $Q_{\imath}$ is the charge flowing into leg $i$, and $\Gamma\left(y_{1} \ldots y_{n}\right)$ is the ordinary $n$-point vertex function, that is the amputated one-particle irreducible part of the Green function $G\left(y_{1} \ldots y_{n}\right)$ defined in Eq. (2.3 b).

The most general conformal invariant Ansatz for the current's 3-point function $G_{u}\left(x ; y_{1} y_{2}\right)$ has already been found by Migdal [1]. For the reader's convenience we reproduce his result.

For a scalar field with charge $Q$ and dynamical dimension $d$

$$
\left\langle T\left\{j^{\mu}\left(x_{3}\right) \phi\left(x_{1}\right) \phi\left(x_{2}\right)\right\}\right\rangle=a_{s s} x_{23}^{-D+2} i \overleftrightarrow{\Gamma}_{3}^{\mu} x_{13}^{-D+2} x_{12}^{D-2-2 d} \Gamma(d) 2^{-d}
$$

while for a Dirac field in $D=4$ space time dimensions

$$
\begin{aligned}
\left\langle T\left\{j^{\mu}\left(x_{3}\right) \psi\left(x_{1}\right) \bar{\psi}\left(x_{2}\right)\right\}\right\rangle= & \sum_{i=S . V, T} a_{i} \Omega_{l} \hat{x}_{23} x_{23}^{-4} \gamma^{\mu} \hat{x}_{31} x_{31}^{-4} \Omega_{l} . \\
& \cdot 2^{d+1 / 2} \Gamma\left(d+\frac{1}{2}\right) x_{12}^{1-2 d} \operatorname{Tr}\left(\Omega_{i} \hat{x}_{12} \Omega_{l} \hat{x}_{12}\right)
\end{aligned}
$$

where

$$
\hat{x}_{i j}=\left(x_{i}-x_{j}\right) \cdot \gamma, \quad x_{i j}^{\alpha}=\left(-\left(x_{i}-x_{j}\right)^{2}+i \varepsilon\right)^{\alpha / 2}, \quad \Omega_{l}=\left(1, \gamma_{x}, \frac{1}{2}\left[\gamma_{i}, \gamma_{\varrho}\right]\right) .
$$

Migdal's selection rule is automatically fulfilled for theories as described at the end of Section 1.

The Ward identities (2.6) are fulfilled if

$$
\begin{gathered}
a_{\mathrm{ss}}=Q \Gamma\left(\frac{1}{2} D-1\right) / 4 \pi^{D / 2} \\
a_{\mathrm{s}}+4 a_{V}+24 a_{T}=Q / 8 \pi^{2} . \quad(D=4) .
\end{gathered}
$$

After Ward identities are imposed, the e.m. vertex of a charged spinor field still depends on 2 parameters, say $a_{V}$ and $a_{T}$. These are not free parameters, however, but are determined by the dynamics of the system.

Indeed, not every conformal invariant expression of the form (2.8) will automatically represent the 3 -point function of a local field $j^{\mu}(x)$. Rather, for this to be the case it is necessary that the appropriate Bethe Salpeter equation be satisfied. It reads

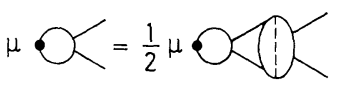

with BS kernel from Eq. (1.3). An inhomogeneous term (bare vertex) is absent, as in Migdal's bootstrap equation (1.1), because it would not be conformal invariant. Summation over charged fields to which the current couples is understood on the RHS of (2.10). Thus, if there is more than one charged field, (2.10) will be a system of equations for the current's various 3-point functions. 
Applying the convergence criteria of Ref. [5] it is straightforward to show that the RHS of Eq. (2.10) is conformal invariant and free from divergences in the context of the present theory.

Thus if we insert into Eq. (2.10) the most general ${ }^{8}$ conformal invariant Ansatz (2.8) for the 3-point functions $G_{\mu}$ the (system of) equation(s) will be thereby solved and one obtains a system of linear homogeneous equations for the coefficients $a_{s s} ; a_{s}, \ldots$. This is not, however, sufficient to guarantee an acceptable solution. For instance, the trivial solution $a_{s s}=\cdots=0$ is not acceptable if $Q \neq 0$ since it does not satisfy the Ward identity condition (2.9). It must, therefore, be shown that also the validity of Ward identities for $G_{\mu}$ is reproduced by the RHS of (2.10). If this is true then Eq. (2.10) reduces to an inhomogeneous system for the parameters (e.g. $a_{V}, a_{T}$ ) that are still free after Ward Takahashi identities have been imposed. Barring unex pected degeneracies it will uniquely determine these parameters. (In the spinless case, such uniqueness is trivial since there is no free parameter left.)

It suffices to verify consistency of the differentiated Ward identity (2.7b), because every conformal invariant expression that satisfies ( $2.7 \mathrm{~b}$ ) will automatically satisfy (2.6) (see last footnote). Basically this comes from the fact that all momentum dependence of 3-point functions is completely determined by conformal invariance.

Let us then insert W.I. (2.7b) on both sides of the BS equation (2.10). Using the notation defined in Eq. (1.2a) with $Q=$ internal charges we find (after amputation)

$$
-x^{-1}=\frac{1}{2} \biguplus_{-\infty}^{*}
$$

It has to be shown that this equation is satisfied for physical values of coupling constants $g$ and dimensions of fields $d$. Physical $g, d$ are such that the bootstrap condition (1.1), (1.2b) are satisfied. Now Eq. (2.11) is another integral equation for the (charged) propagators. We shall show in Section 4 that it is equivalent to Eq. (1.2b) (with $Q=$ internal charge) modulo validity of Migdal's vertex bootstrap condition (1.1). Thus it will indeed be satisfied if Eqs. (1.1) and (1.2b) are true. Conversely we may also use (2.11) in place of Eq. (1.2b) as propagator bootstrap. Of course, this is true only for a charged propagator. We shall however see in Section 3 that there is an analogous equation coming from the Ward identity requirement for the stress energy tensor, which can be used also for purely neutral fields.

8 Conservation of the 3-point Wightman functions (but not, of course, correct normalization!) turns out to be an automatic consequence of conformal symmetry and canonical dimensionality $d=D-1$ of the current. Here as everywhere $D=$ No. of space time dimensions ( 4 in the real world). 
With the current's 3-point function determined, the higher $n+1$-point vertex functions, $n \geqq 3$ can be constructed by skeleton expansion:

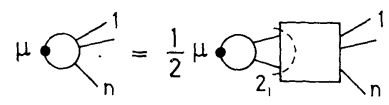

We have drawn a box instead of a bubble to indicate that only the whole graphs need be connected and one-particle irreducible. This notation is explained in Appendix A.

It will be shown in Section 4 that all the Green functions abtained from this construction satisfy Ward Takahashi identities (2.7c).

Amplitudes involving several currents may be constructed in the same way. (cp. e.g., Sections I.5 and VI.3 of Ref. [27]) e.g., the vertex functions involving two currents and one or more field operators are calculated from

$$
\sum_{1}^{n}=\frac{1}{4}
$$

and are finite for noncoinciding arguments, by the criteria of Ref. [5]. A new feature appears, however, when one constructs Green functions involving only currents - i.e. fields of canonical (integer) dimensionality and no other fields.

Let us consider as an example the 2-point function

$$
G_{\varrho \sigma}(x, 0)=\left\langle 0\left|T^{*}\left\{j_{\varrho}(x) j_{\sigma}(0)\right\}\right| 0\right\rangle .
$$

It can be computed according to (cp. Section VI.3 of Ref. [27])

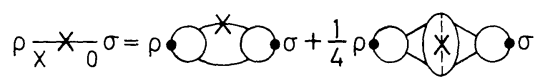

with the "momentum differentiated" BS kernel given by Eqs. (1.2a), or (1.4), where $Q=P^{\mu}$ a component of 4-momentum. (To work out the first term one needs the amputated vertex function. Amputation can be readily performed on Eq. (2.8) with the help of the "vertex graph identity" of Parisi, Peliti and d'Eramo [28].)

The RHS of (2.15) turns out to be well-defined and dilatation invariant for $x \neq 0$. (Recall that overall divergence in momentum space does not show up in configuration space for $x \neq 0$.) Because of current conservation (implied by the results of Section 4) the result must, therefore, come out in the form

$$
G_{\mu \nu}(x, 0)=\operatorname{const}\left(\nabla_{\mu} \nabla_{v}-g_{\mu \nu} \square\right)\left(-x^{2}+i 0\right)^{-D+2}, \quad x \neq 0 .
$$

This function does not posses an extension to a conformal invariant (or even dilatation invariant) distribution if $D=$ even integer $\geqq 2$. This is the 
"vengeance of canonical dimensionality". The most general extension of expression (2.16) to a distribution is

$$
\operatorname{const}\left(\nabla_{\mu} \nabla_{v}-g_{\mu v} \square\right)\left(-x^{2}+i 0\right)^{-D+2}+\Sigma C_{n} \square^{n} \delta(x) .
$$

The natural choice is to set the $C_{n}$ with $n>\frac{1}{2} D-2$ equal to zero. In (2.17) the first term is now defined by

$$
\left(-x^{2}+i 0\right)^{-D+2}=\lim _{\alpha \rightarrow-D+2} \frac{\partial}{i \alpha}(\alpha+D-2)\left(-x^{2}+i 0\right)^{\alpha} .
$$

This definition is appropriate because the distribution $\left(-x^{2}+i 0\right)^{x}$ has a pole at $\alpha=-D+2$ with residue proportional $\square^{\frac{1}{2} D-2} \delta(x)$. Expression (2.18) is not a homogeneous distribution - its Fouriertransform contains a factor $\log q^{2}$. This means that it is impossible to define the $T^{*}$-product in (2.14) in such a way that it is dilatation invariant. This is a special feature associated with canonical dimensionality.

Let us note, however, that the Wightman function of two currents is dilatation and conformal invariant. It is uniquely determined by (2.16) and analyticity requirements in configuration space:

$$
\left\langle 0\left|j_{\mu}(x) j_{v}(0)\right| 0\right\rangle=\mathrm{const}\left(\nabla_{\mu} \nabla_{v}-g_{\mu v} \square\right)\left(-x^{2}+i \varepsilon x^{0}\right)^{-D+2} .
$$

In contrast with expression (2.17) this is a well defined homogeneous distribution. As a consequence the well known prediction [29] of dilatation symmetry for the total cross section of $e^{+} e^{-} \rightarrow$ hadrons is true in the present theory without modification. Even its absolute magnitude could be computed in principle. To this end one would have to evaluate and sum the graphs contributing to the RHS of Eq. (2.15).

\section{The Stress Energy Tensor}

In a local quantum field theory there ought to exist a conserved stress energy tensor $\theta_{\mu \nu}$ such that generators

$$
P_{\mu}=\int d \sigma^{\varrho} \theta_{\varrho \mu}(x), \quad M_{\mu v}=\int d \sigma^{\varrho}\left(x_{\mu} \theta_{\varrho v}-x_{v} \theta_{\varrho \mu}\right)
$$

implement infinitesimal Poincaré transformations, viz.

$$
\left[\phi(x), P^{\mu}\right]=i \nabla^{\mu} \phi(x) ; \text { etc. }
$$

In a conformal invariant theory one shall also want the stress tensor to implement infinitesimal dilatations and special conformal transformations. That is, currents

$$
D_{v}(x)=x^{\varrho} \theta_{v \varrho} ; \quad K_{v \mu}(x)=2 x^{\varrho} x_{\mu} \theta_{v \varrho}-x^{2} \theta_{v \mu}
$$


ought to be conserved and obey commutation relations

$$
\begin{array}{rlrl}
\int_{\Omega} d \sigma_{x}^{v}\left[\phi(0), D_{v}(x)\right]_{-} & =-\mathrm{id} \phi(0) & \\
\int_{\Omega} d \sigma_{x}^{v}\left[\phi(0), K_{v \mu}(x)\right]_{-} & =0 & 0 \in \Omega
\end{array}
$$

with $d$ the dynamical dimension of mass of the field $\phi$. Conservation of currents $(3.3 \mathrm{a})$ requires that the stress tensor be traceless:

$$
\theta_{\mu}^{\mu}(x)=0 .
$$

Wilson [12] has shown that the stress tensor must necessarily have dimension $d_{\theta}=D$.

For the sake of clarity it is convenient to consider first the 3-point Wightman function

$$
W_{\mu v}\left(x ; y_{1}, y_{2}\right)=\left\langle 0\left|\theta_{\mu v}(x) \phi\left(y_{1}\right) \phi\left(y_{2}\right)\right| 0\right\rangle .
$$

It must be conserved, symmetric and traceless.

$$
\text { (a) } \nabla_{x}^{\mu} W_{\mu v}\left(x ; y_{1}, y_{2}\right)=0 ; \quad \text { (b) } W_{\mu}^{\mu}\left(x ; y_{1}, y_{2}\right)=0 \text {. }
$$

Constructing the most general conformal invariant Ansatz satisfying these requirements one finds first of all the selection rule

$$
\left\langle 0\left|\theta_{\mu \nu}(x) \phi(y) \psi(z)\right| 0\right\rangle=0 \quad \text { if } \quad d_{\varphi} \neq d_{\psi} .
$$

For the nonvanishing 3-point functions it turns out that tracelessness (3.6b) plus conformal invariance with $d_{\theta}=D$ implies conservation (3.6a) already. (See note added in proof.)

Let us first consider scalar fields $\phi(x)$ only: The most general traceless conformal invariant Ansatz is found to be (cp., e.g., Ref. [30])

$$
\begin{aligned}
W^{\mu v}\left(x_{1} ; x_{2}, x_{3}\right)= & b_{s} x_{12}^{-D+2}\left[\bar{\nabla}_{1}^{\mu} \vec{\nabla}_{1}^{v}+\bar{\nabla}_{1}^{v} \vec{\nabla}_{1}^{\mu}-g^{\mu v} \bar{\nabla}_{1}^{o} \vec{\nabla}_{\varrho 1}\right. \\
& \left.+\frac{D-2}{2(D-1)}\left(g^{\mu v} \tilde{\nabla}_{1}^{o} \tilde{\nabla}_{\varrho 1}-\tilde{\nabla}_{1}^{\mu} \tilde{\nabla}_{1}^{v}\right)\right] x_{13}^{-D+2} x_{23}^{D-2-2 d}
\end{aligned}
$$

in a world with $D$ space time dimensions. Here

$$
x_{i j}^{\alpha}=\left[-\left(x_{i}-x_{j}\right)^{2}+i \varepsilon\left(x_{i}^{0}-x_{j}^{0}\right)\right]^{\alpha / 2} ; \quad \tilde{\nabla} \equiv \vec{\nabla}+\bar{\nabla} .
$$

The Wightman function corresponding to a different ordering of fields is obtained from (3.8) by analytic continuation in the coordinates $x_{i}$. In particular it is therefore possible to compute from (3.8) equal-time commutators. Recalling Eq. (1.5) one finds

$$
\left.\left\langle 0\left|\left[\theta^{0 v}(x), \phi(y)\right] \phi(z)\right| 0\right\rangle\right|_{x^{0}=y^{0}}=-i \Gamma_{v}\langle 0|\phi(y) \phi(z)| 0\rangle \delta(\mathbf{x}-\mathbf{y})+\cdots
$$


provided one chooses

$$
b_{s}=\frac{1}{4} \pi^{-\frac{1}{2} D} \Gamma\left(\frac{1}{2} D\right)^{-1} \Gamma(1+d) 2^{d} .
$$

To verify this one uses the well known identity

for

$$
\left.\frac{\partial}{\partial x^{0}} \Delta(x)\right|_{x^{0}=0}=-\delta(\mathbf{x})
$$

$$
\Delta(x)=\frac{1}{4} i \pi^{-D / 2} \Gamma\left(\frac{1}{2} D-1\right)\left\{\left(-x^{2}+i \varepsilon x^{0}\right)^{-\frac{1}{2} D+1}-\text { c.c. }\right\} .
$$

In Eq. (3.10) the dots stand for gradient terms. The implications of C.R. (3.2) for the 2-point function are, therefore, fulfilled. The C.R. of the remaining generators $M_{\mu \nu}, D, K_{\mu}$ could also be verified by completing Eq. (3.10).

The time ordered Green function

$$
G^{\mu v}\left(x, y_{1} y_{2}\right)=\left\langle 0\left|T^{*}\left\{\theta^{\mu v}(x) \phi\left(y_{1}\right) \phi\left(y_{2}\right)\right\}\right| 0\right\rangle
$$

is determined by the Wightman function up to contact terms popularly known as "seagulls". We shall wish to choose these seagulls such that the $T^{*}$ product is conformal invariant; this fixes them up to one free constant $\lambda$. The result is

$$
\begin{aligned}
G_{\mu v}\left(x_{1} ; x_{2} x_{3}\right)= & \text { RHS of }(3.8)+\frac{\lambda}{4 \pi^{D / 2}} \Gamma\left(\frac{D}{2}-1\right) b_{s} \\
& \cdot\left\{\delta\left(x_{1}-x_{2}\right)+\delta\left(x_{1}-x_{3}\right)\right\} x_{23}^{-2 d} g_{\mu v}
\end{aligned}
$$

but with a changed $i \varepsilon$-prescription everywhere, viz.

$$
x_{i j}^{\alpha}=\left[-\left(x_{i}-x_{j}\right)^{2}+i \varepsilon\right]^{\alpha / 2} .
$$

From expression (3.13) one obtains the Ward Takahashi identity

$$
\begin{gathered}
\nabla_{x}^{\mu} G_{\mu v}\left(x, y_{1} y_{2}\right)=-i\left(\delta\left(x-y_{1}\right) \vec{\nabla}_{1}^{v}+\delta\left(x-y_{2}\right) \vec{V}_{2}^{v}\right) G\left(y_{1} y_{2}\right) \\
-i \lambda \nabla_{x}^{v}\left\{\delta\left(x-y_{1}\right)+\delta\left(x-y_{2}\right)\right\} G\left(y_{1} y_{2}\right) \cdot 2 d(D-2)^{-1}
\end{gathered}
$$

with 2-point function $G\left(y_{1} y_{2}\right)$ given by Eq. (1.5).

We shall find it convenient later to specify $\lambda$ such that the Green function is traceless:

$$
G_{\mu}^{\mu}\left(x ; y_{1}, y_{2}\right)=0 .
$$

This is achieved by taking

$$
\lambda=(2-D) / 2 D .
$$

With this choice of $\lambda$ there remains in (3.15) the "abnormal" second term. We emphasize, however, that Ward identities (3.15) do guarantee correct 
commutation relations (3.2), (3.3) as is clear from our discussion of the Wightman function.

Not every conformal invariant Ansatz for the 3-point function $G_{\mu v}$ need a priori be matrix element of a local field $\theta_{\mu v}(x)$. Rather, the 3-point function should also fulfill the appropriate Bethe-Salpeter equation viz.

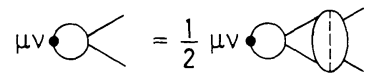

On the RHS, summation is understood over the fields to which the stress tensor couples (i.e. all of them).

We must show first that the RHS of (3.18) is finite, if so it will then automatically be conformal invariant by the results of Ref. [5].

We recall that conformal invariant skeleton graphs may be represented by generalized Feynman integrals in the sense of Speer [31]; this is done by substituting the graphical representation of the dressed 3-point vertex, Fig. 1, where undotted lines stand for dressed propagators,

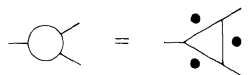

Fig. 1. The dressed vertex

Eq. (1.5), while dotted lines stand for generalized Feynman propagators $2^{d^{\prime}} \Gamma\left(d^{\prime}\right)\left(-x^{2}+i 0\right)^{-d^{\prime}}$ with $d^{\prime}$ such that at every "bare vertex" one has conservation of dimension $\Sigma d=D$. Since overall divergences are harmless as discussed before, we only have to look for divergent proper subgraphs. There are two of them; they are shown in Fig. 2. They are found

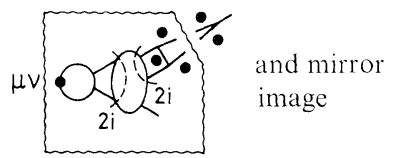

Fig. 2. Divergent subgraphs in RHS of Eq. (3.18)

to be logarithmically divergent for arbitrary values of the dynamical dimension $d$. However, the divergent part of a logarithmically divergent generalized Feynman integral is independent of momenta. Therefore, by Lorentz invariance it must be proportional to $g^{\mu \nu}$, as $g^{\mu v}$ is the only symmetric $2^{\text {nd }}$ rank tensor that can be formed with no momenta available. (This argument still holds with spin $\frac{1}{2}$ fields present, since $\left\{\gamma_{\mu}, \gamma_{\nu}\right\}=2 g_{\mu \nu}$.) Thus a divergence could only arise in the trace. However, the integrand is traceless by (3.16), therefore the RHS of (3.18) is also traceless and free from divergences ${ }^{9}$.

9 The reader will now understand why we chose seagulls such that $G_{\mu \nu}$ is traceless, as it would be with an ordinary $T$-product. 
Thus (3.18) is a meaningful equation. We still have to show that it is fulfilled by the Ansatz (3.13) with $b_{s}$ from (3.11), for physical values of coupling constant $g$ and dimension $d$. As in the analogous case of internal symmetry currents it suffices to show that the Ward identity at zero momentum transfer is correctly reproduced by the equation.

Let us use the following notation

$$
\left.\begin{array}{c}
x \\
\frac{x}{x_{i}} x_{f}^{-1}
\end{array}\right\}=P^{v}\left(x_{i}^{\sigma}-x_{f}^{\sigma}\right)-\frac{1}{D} g^{v \sigma} P_{\tau}\left(x_{i}^{\tau}-x_{f}^{\tau}\right)\left\{\begin{array}{l}
- \\
-1
\end{array}\right.
$$

where $P$ is the momentum flowing from $i$ to $f$. In configuration space language $P^{\mu}=-i \nabla_{x}^{\mu}$. Definition (3.19a) agrees with (1.2a) for $\mu \neq v$. Substituting WTI (3.15) on both sides of Eq. (3.18) and integrating over $\int d x x^{\sigma}$ we obtain (after amputating)

$$
-x^{-1}=\frac{1}{2} \leftrightarrows
$$

It suffices to consider this equation for $v \neq \sigma$ in (3.19a). The validity for the traceless part then follows from Lorentz invariance.

Eq. (3.19b) is the analog of Eq. (2.11), with the external charge replaced by momentum in ( 3.19 a). Noncommutativity of $P, x$ is irrelevant here since $\left[P_{v}, x_{\sigma}\right]=0$ for $v \neq \sigma$.

We shall show in Section 5 that Eq. (3.19b) is equivalent to imposing unitarity on the 2-point functions. We shall also show, in Section 5, that it is equivalent to the "momentum form" of Eq. (1.2b). Thus it will be satisfied if the bootstrap conditions are fulfilled. Alternatively one may use ( $3.19 \mathrm{~b}$ ) itself as the propagator bootstrap, all the other propagator equations (1.2b), (2.11) and propagator unitarity are then automatically fulfilled, modulo validity of the vertex bootstrap (1.1).

In a theory with spin $\frac{1}{2}$ fields, the most general Ansatz for the stress tensor's 3-point function involves some arbitrary coefficients, much as in Eq. (2.8) for the current. They have to be determined from Eq. (3.18) in analogy with the procedure described in Section 2 for the current.

With the stress tensor's 3-point function known, the higher $n$-point functions may again be constructed by skeleton graph expansion as for the internal symmetry currents in (2.12), viz.

$$
\mu \vee \mathcal{F}=\frac{1}{2} \mu \vee \bigcirc
$$

The result is finite and conformal invariant by the criteria of Ref. [5]. It will be shown in Section 4 that the construction will also guarantee validity of Ward Takahashi identities. 
Written out for vertex functions they read

$$
\begin{aligned}
\nabla_{x}^{\mu} \Gamma_{\mu \nu}\left(x ; y_{1} \ldots y_{n-1}\right)= & -i \sum_{k=1}^{n-1}\left\{\delta\left(x-y_{k}\right) \vec{\nabla}_{\lambda v} \Gamma\left(y_{1} \ldots \hat{y}_{k} \ldots y_{n-1} x\right)\right. \\
& \left.+\left(\frac{d}{D}-1\right) \vec{V}_{\lambda v}\left[\delta\left(x-y_{k}\right) \Gamma\left(y_{1} \ldots \hat{y}_{k} \ldots y_{n-1} x\right)\right]\right\} .
\end{aligned}
$$

This is equivalent (cp. Section I.5 and II.3 of Ref. [27]) to WTI for Green functions which read in functional form

$$
\nabla_{\mu} G^{\mu v}(x, J\}=-i J(x) \vec{\nabla}_{x}^{v} G_{x}\{J\}+i \frac{d}{D} \vec{\nabla}_{x}^{v}\left(J(x) G_{x}\{J\}\right) .
$$

The notation used here is analogous to that defined in (2.3) and following equations.

\section{Verification of Ward Identities}

In this section we shall demonstrate that

a) The $n$-point functions $(n \geqq 4)$ constructed by skeleton graph expansions (2.12) resp. (3.20) satisfy Ward Takahashi identities as are required by current conservation and C.R. (2.2) resp. (3.2) f.

b) Eqs. (2.11) resp. (3.19) are equivalent to the internal symmetry resp. momentum form of the propagator bootstrap $(1.2 \mathrm{~b})$.

Both of these statements hold only if the vertex bootstrap condition (1.1) is fulfilled.

Let us start with the WTI for internal symmetry currents. We shall use the following notation, which generalizes (1.2a). Let $\varphi(x)$ a test function. Then, for a general $n$-point vertex, we define

$$
\stackrel{\varphi}{X}_{x_{n}}^{x_{1}}=\sum_{i=1}^{n} Q_{1} \varphi\left(x_{1}\right) \mathcal{F}_{x_{n}}^{x_{1}} \quad Q_{i}=\text { charge flowing into leg } i
$$

In particular $\quad \otimes^{\varphi} \equiv D_{v}^{\varphi} \bigcirc=\sum_{i}^{3} Q_{i} \varphi\left(x_{i}\right) \bigcirc$

and

$\frac{\varphi}{X_{1}} X_{X_{f}}=D_{l}^{\varphi}-=Q\left[\varphi\left(X_{i}\right)-\varphi\left(X_{f}\right)\right]-; \quad Q=$ charge flowing from $i$ to $f$.

If we insert the WTI (2.6) for the 3-point function $G_{\mu}\left(x ; y_{1}, y_{2}\right)$ into the RHS of skeleton graph expansion (2.12) we see that validity of WTI- 
identities ( $2.7 \mathrm{c})$ for $n+1 \geqq 4$ point functions holds if

$$
\frac{1}{2} \varphi \gtrless_{2 i} \mathbb{F}_{n}^{\prime}=\stackrel{\varphi}{\times} \Psi_{n}^{\prime}
$$

for arbitrary test function $\varphi$. As always, the quadratic box is used to indicate that (connectedness and) 1-particle irreducibility requirements are waived, but only to the extent that the whole graph remains connected and 1-particle irreducible (see Appendix A).

We will begin with deriving Eq. (4.3) for $n=3$ from the vertex bootstrap (1.1). Let us note that (1.1) may be written as

$$
-Q=\sum_{\Gamma}-\mathbb{C}
$$

Summation is over all nontrivial skeleton graphs $\Gamma$. The skeleton condition means in this case that there is no proper 3-point subgraph, and no selfenergy subgraph.

Next we observe that the $\stackrel{\varphi}{\times}$-operation defined in (4.1) may be distributed over any skeleton graph in an obvious way, as explained after Eq. (1.4). Because of charge conservation all terms involving $Q_{i} \varphi\left(x_{i}\right)$, with $x_{l}$ an integration variable, will cancel out. We obtain in this way from Eq. (1.1')

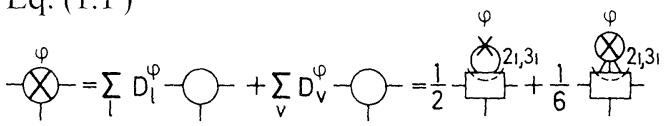

The irreducibility requirements $2 i, 3 i$ are explained in Appendix A. They come from the skeleton condition on $\left(1.1^{\prime}\right)$. The meaning of boxes was explained after Eq. (4.3). We now consider Eq. (4.4) as a linear inhomogeneous integral equation for the "crossed" vertex. Its solution, if one exists, will be unique, assuming that the homogeneous equation has no nontrivial solution. This will be ruled out below. Thus it suffices to demonstrate that expression (4.3) for $n=3$ is a solution of (4.4). Inserting it gives

$$
\frac{1}{2}=\frac{1}{2}
$$

Both sides of this equation involve exactly the same set of skeleton graphs, thus equality is manifestly true.

Let us now discuss the homogeneous equation associated with (4.4). Since there is no reason to suspect that it has a nontrivial solution in the first place, we shall be content with very briefly sketching an argument.

To arrive at Eq. (4.4) we have only used the vertex bootstrap (1.1). The vertex bootstrap determines the dynamical dimension(s) $d$ as power 
series ${ }^{10}$ in $g$, but still leaves free the coupling constant $g$. To take advantage of this fact it is convenient to consider temporarily the Green functions defined by skeleton expansion for arbitrary values of $g$, with the understanding that the propagator bootstrap is to be imposed only at the end to fix $g$, while the dimensions have already been determined as functions of $g$ through the vertex bootstrap. Eq. (4.4) is then an identity in $g$, and both sides are power series in $g$. (For $\phi^{3}$-type theory this follows from holomorphy of the RHS of (4.4) in $d$. For Yukawa theory a slightly more involved argument can be given [32].) Now the difference of RHS and LHS of Eq. (4.3) would have to be a solution of the homogeneous equation associated with (4.4). On the other hand (by the same argument as above) it is a power series in $g$, even after dependence of $d$ on $g$, i.e. the vertex bootstrap, has been taken into account. But a nontrivial power series solution of the homogeneous equation (4.4) obviously does not exist.

It may be instructive for the reader to remark that Eq. (4.3) for $n=3$ may also be obtained from Eq. (4.4) by iteration, assuming the iteration converges. After the first iteration step

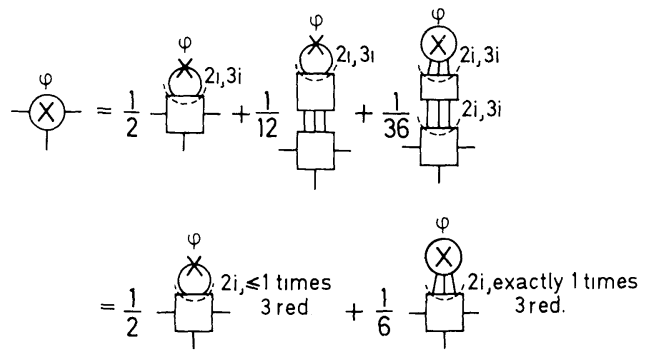

and after $N$ iteration steps

$$
\underbrace{\varphi}_{-1}=\frac{1}{2}
$$

We must now assume that the iteration converges, that is the second term on the RHS tends to zero as $N \rightarrow \infty$. Then we obtain relation (4.3) for $n=3$ by letting $N \rightarrow \infty$ in Eq. $\left(4.5^{\prime \prime}\right)$. In particular, if we specialize to $\varphi(x)=x^{v}$ we obtain

$$
x_{1}-\underset{x_{2}}{\bigotimes}-x_{3}=\frac{1}{2} x_{1}-\underbrace{-y_{1}^{2 i}}_{x_{2}} x_{3}
$$

in the notation of Eq. (1.2a), $Q=$ internal charge (see Appendix B).

${ }^{10}$ This follows from the holomorphy properties of the RHS of Eq. (1.1) in dimensions $d$, which are implicit in Ref. [5]. 
With relation (4.3) proven for $n=3$ we can use this result to establish its validity also for $n \geqq 4$. We start from the skeleton graph expansion for the ordinary $n$-point vertex function. We apply the operation $\stackrel{\varphi}{\times}$ and distribute the cross over each skeleton graph by summing over all possibilities of attaching the cross to a line or vertex, as before. We obtain

$$
\begin{aligned}
& \mathbb{X}_{n}^{\varphi}=\sum_{1} D_{1}^{\varphi}, Q_{n}+\sum_{v} D_{v}^{\varphi}, Q_{n}=
\end{aligned}
$$

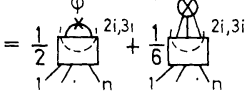

as a generalization of (4.4). We now insert Eq. (4.3) for $n=3$ to obtain

$$
\text { (47) }=\frac{1}{2} \underbrace{21,31}_{n}+\frac{1}{12}, \underbrace{21,31}_{n}=\frac{1}{2}
$$

q.e.d. Note that the LHS of Eq. (4.3) includes some graphs with 3-point subgraphs; these 3-point subgraphs contain the line with cross attached, however. An example is shown in Fig. 3.

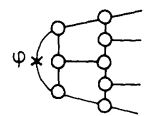

Fig. 3. A graph contained in the LHS of Eq. (4.3)

This type of contribution is provided by the second term on the RHS of Eq. (4.7) or (4.8). (For a pertinent remark hereto, see the end of Appendix B.)

Finally we wish to show that Eq. (2.11) is equivalent to the internal charge form of the propagator bootstrap (1.2b).

Let us consider the RHS of Eq. (1.2b) and distribute the $\times$-operation as in Eq. (1.4). That is

$$
\begin{aligned}
& X=\sum_{e} D_{e} Q+\sum_{v} D_{v} \backslash=
\end{aligned}
$$

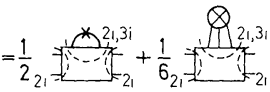

with notation as in Eq. (1.2a). (No superscript $\varphi$ means $\varphi(x)=x^{v}$.) Inserting identity (4.6) gives

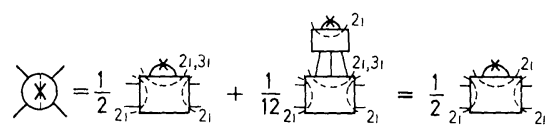


Thus finally

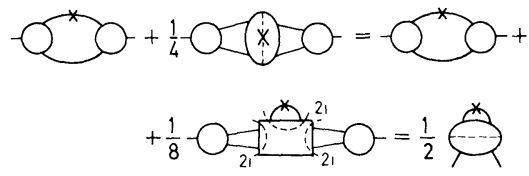

q.e.d.

Let us now turn to the stress energy tensor. Let us introduce the notation

$\frac{\varphi}{x_{1} x_{2}}=\left[\varphi\left(x_{1}\right) \vec{\nabla}_{1}^{v}+\varphi\left(x_{2}\right) \vec{\nabla}_{2}^{v}-\frac{d}{D} \varphi^{, v}\left(x_{1}\right)-\frac{d}{D} \varphi^{\cdot v}\left(x_{2}\right)\right]_{x_{1} \quad x_{2}}$

with $\varphi^{v}(x)=\nabla_{x}^{v} \varphi(x)$ and $d$ the dimension of the field. Let further

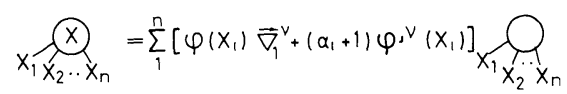

with $\alpha_{i}$ to be determined below.

Now consider

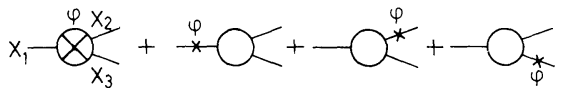

which may be considered as part of a larger graph. We will determine $\alpha_{i}$ in such a way that all contributions involving $\varphi\left(x_{i}\right)$ or $\varphi_{, v}\left(x_{i}\right)$ for $i=1,2,3$ combine to a total derivative which drops out after integrating over $x_{1}, x_{2}, x_{3}$. One finds by straight-forward computation that this is satisfied if one chooses

$$
\alpha_{i}=\frac{1}{D} d_{i}
$$

$d_{i}=$ dynamical dimension of the field in the propagator attached to $x_{t}$.

With these conventions, the $\stackrel{\varphi}{x}$-operation may again be distributed over any graph as in the treatment of internal-symmetry currents. Contributions involving $\varphi\left(x_{i}\right)$ or $\varphi_{, v}\left(x_{i}\right)$, with $x_{i}$ an integration variable, drop out after integration over $x_{i}$. Thus from here on we may repeat word for word the discussion of Ward identities for internal symmetries. We find in this way that

a) WTI (4.3) hold also for the stress energy tensor, with operation $\stackrel{\varphi}{\times}$ explained in Eqs. (4.11).

b) Eq. (3.19) is equivalent to the momentum form of the propagator bootstrap (1.2b).

Part b) could also have been proven without going through $(4.11 \mathrm{c})$ by noting that $\varphi_{, v}=0$ if $\varphi(x)=x^{\mu}, \mu \neq v$. 
The Ward identities (4.3) imply that WTI (3.21) are fulfilled. To see this one takes the divergence of skeleton expansion (3.20) and inserts the WTI for the 3-point function on the RHS. After smearing with a testfunction $\varphi$ this can be combined with Eq. (4.3) to imply (3.21).

The proof of Ward identities remains valid as it stands if there are two or more currents involved in the Green function. Let $j_{v}(x)$ the current with respect to whose argument one wants to differentiate. It is then always possible to choose linear combinations $j_{\mu}^{a}\left(x^{\prime}\right)$ of currents such that

$$
\left[j_{\mu}^{a}\left(x^{\prime}\right), j_{0}(x)\right]_{\mathrm{e} . \mathrm{t} .}=q^{a} j_{\mu}^{a}\left(x^{\prime}\right) \delta\left(\mathbf{x}^{\prime}-\mathbf{x}\right)+\text { S.T. },
$$

as for the fundamental fields discussed in Section 2. The vertex functions involving several currents are constructed by skeleton expansion as in Eq. (2.13). To verify the Ward identities, one first performs the cross operation on (2.10) and solves for the crossed three-point function as (4.4) was solved for the crossed vertex. The result is

$$
\dot{x}=\sum_{n}^{\mu}+\frac{1}{2} \sum_{-\rightarrow}^{\mu}+\frac{1}{4} \underbrace{\mu}_{\frac{-x}{n}}
$$

where the box is the four-point vertex function plus the two skeleton graphs one-particle reducible in the crossed channels. Taking the divergence of (2.13) gives, using (2.12).

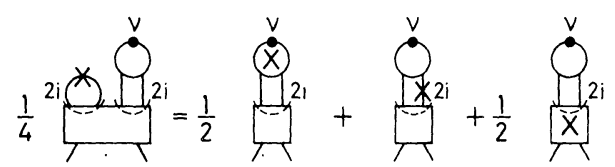

as desired, equality being proven with the help of (4.13), (4.9) and (4.3) in the forms

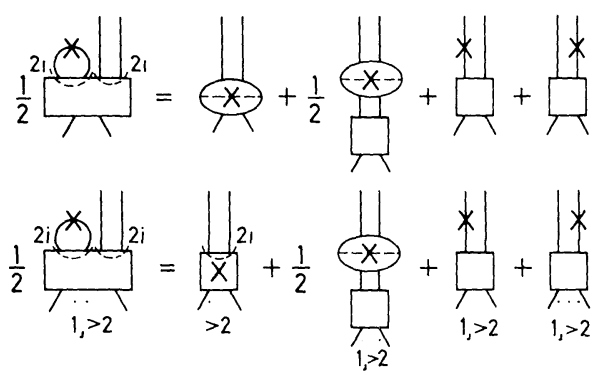

The generalized Ward identities (4.3) have an interesting geometrical interpretation. They reflect local implementability of non-symmetry gauge 
transformations resp. space time transformations. For instance, the Ward identity for $\theta_{\mu \nu}$ at zero momentum transfer (i.e. with $\varphi(x)=x^{\nu}$ ) corresponds to a transformation $x^{\alpha} \rightarrow x^{\alpha}+\varepsilon a_{\beta}^{\alpha} x^{\beta}$ where $a_{\alpha}^{\alpha}=0$ but $a^{\alpha \beta} \neq-a^{\beta \gamma}$. (A different type of such transformation - an infinitesimal change in physical coupling constant - will be discussed in Appendix C. Its local implementability is related to causality, cf. Ref. [33].) Thanks to the tracelessness of $G^{\mu v},(3.16)$, the cross operations to all infinitesimal symmetry transformations (i.e., to elements of the full conformal group) give zero identically.

\section{Unitarity}

We recall first that the contribution of any skeleton graph in conformal invariant field theory may be written as a generalized Feynman integral in the sense of Speer [31]. In other words, the integrand is a product of generalized Feynman propagators

$$
\Delta_{F}\left(x_{i j}, \dot{\delta}_{i j}\right)=2^{\delta_{1 \jmath}} \Gamma\left(\delta_{i j}\right)\left(-x_{i j}^{2}+i \varepsilon\right)^{-\delta_{\imath \jmath}} ; \quad x_{i j}=x_{i}-x_{j}
$$

with certain prescribed values of exponents $\delta_{i j}$. Indeed, the dressed propagator is itself of this form, and the dressed vertex function may be written as a product of 3 of them, as indicated in Fig. 1.

We observe that such generalized propagators may be decomposed as

$$
\Delta_{F}(x, \delta)=\theta\left(x^{0}\right) \Delta^{+}(x, \delta)+\theta\left(-x^{0}\right) \Delta^{-}(x, \delta)
$$

where $\Delta^{+}$is a positive frequency function

$$
\Delta^{+}(x, \delta)=2^{\delta} \Gamma(\delta)\left(-x^{2}+i \varepsilon x^{0}\right)^{-\delta} \propto \int_{p^{2}>0} d p e^{-i p x} \theta\left(p^{0}\right)\left(p^{2}\right)^{-\frac{1}{2} D+\delta}
$$

and $\Delta^{-}(x, \delta)$ is a negative frequency function.

As a consequence, the hypothesis of Veltman's general cutting rule [34] is satisfied ${ }^{11}$. This gives a rule for computing the absorptive parts (discontinuties) of an arbitrary generalized Feynman integral. The result is not yet of the form of an off-mass-shell unitarity relation for our skeleton theory, however, because one also has to admit for cuts through dressed 3-point vertices. They come from cuts through dotted lines in the notation of Fig. 1 and Ref. [5], viz.

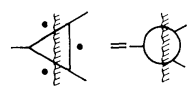

The off-mass-shell unitarity relation suitable for a zero mass infraparticle theory involves only cuts through physical dressed propagator lines,

11 We are referring here to Section 2 of Veltman's paper; its main result Eq. (2.10) and following paragraph will be referred to as "general" cutting formula, to distinguish it from formulae discussed in later sections of Veltman's paper. 
i.e. undotted lines. It reads

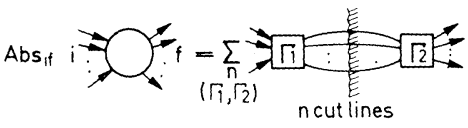

Here, a cut line stands for the positive-frequency absorptive part of a dressed propagator. As a consequence, only positive energy can flow from the unshaded to the shaded side of the cut. Complex conjugation is understood for the part of the graph on the shaded side of the cut. Summation is over pairs of skeleton graphs $\left(\Gamma_{1}, \Gamma_{2}\right)$ such that the entire graph (with the cut imagined absent) does not contain any selfenergy subgraphs. Also, connectedness is only required for the graph as a whole, i.e. each half of the graph may be composed of several disconnected pieces, among them even single lines. Cuts through external lines are understood to have no effect on vertex functions.

The form (5.3) of the unitarity relation, which involves absorptive parts of dressed propagators, rather than free ones (phase space) was discussed by Polyak ov [22] and other authors.

To prove validity of relation (5.3) in our theory, let us consider first the 3-point vertex. Veltman's general cutting formula gives

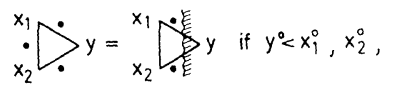

which is also obvious by inspection. It also gives ${ }^{12}$

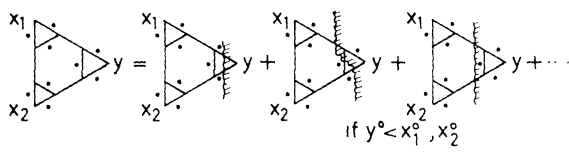

and analogous equations for the higher order graphs in the vertex bootstrap equation (1.1) or (1.1'). Now on account of the vertex bootstrap, the LHS of Eq. (5.4a) agrees with that of (5.4b) plus higher ones for all $y$, $x_{1}, x_{2}$ and therefore in particular for $y^{0}<x_{1}^{0}, x_{2}^{0}$. Thus the right hand sides, which are the analytic continuation of the left hand sides from $y^{0}<x_{1}^{0}, x_{2}^{0}$ through the upper $y^{0}$ half plane to all real $y^{0}$, must also agree for all $y, x_{1}, x_{2}$ by uniqueness of analytic continuation. Analyticity in the upper $y^{0}$ half plane is true because only positive energy can flow through the cut.

${ }^{12}$ Here $x_{1}, x_{2}$ are unmarked " $x$-type" vertices in Veltman's nomenclature, while $y$ and all internal vertices are " $y$-type" vertices. Veltman's alternating signs have been absorbed into factors $-i$ resp. $i$ for each marked resp. unmarked internal vertex. If $y$ is unmarked, no internal vertex can be marked for frequency reasons. 
Summing up, we have in obvious graphical notation, for unrestricted external arguments (internal ones are integrated over)

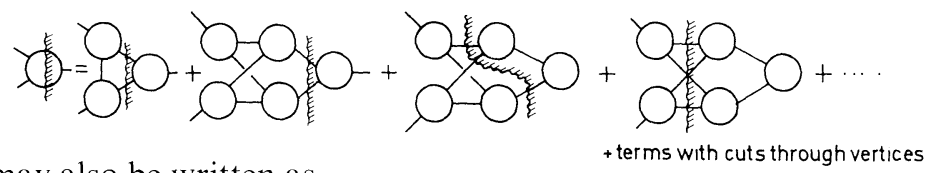

This may also be written as

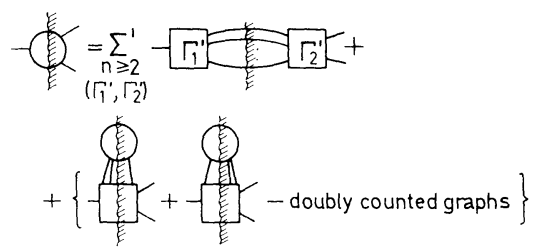

There may be further cut vertices inside the boxes in the second term. Summation in the first term is only over pairs $\left(\Gamma_{1}^{\prime}, \Gamma_{2}^{\prime}\right)$ of skeleton graphs such that the entire graph contains neither a selfenergy subgraph, nor a proper subgraph with 3 external legs. Eq. (5.4c) is an integral equation for the cut vertex. We note that it is solved by the unitarity relation (5.3) for the 3-point function: Upon inserting this Ansatz, the first term in (5.4c) reproduces those terms on the RHS of (5.3) which do not contain 3 -point subgraphs. This is not yet the whole of the RHS of (5.3) because only selfenergy subgraphs were forbidden there. The remaining contributions which contain cut 3-point subgraphs are reproduced by the terms in \{\} on the RHS of (5.4c). An example of such a graph is shown in Fig. 4.

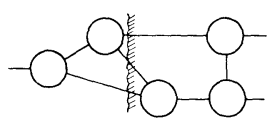

Fig. 4. The simplest graph on the RHS of (5.3) that is not included in the first term on RHS of $(5.4 \mathrm{c})$

Thus we can be sure that generalized unitarity (5.3) is satisfied for the 3 -point vertex if we know that the solution of integral equation (5.4c) for the cut vertex is unique. For this it is sufficient to assume that it can be solved by iteration. One can also verify directly that unitary relation (5.3) for the 3-point vertex is recovered by iteration of Eq. (5.4c). The iteration will converge if the contribution of certain $N$ times 3-particle reducible graphs tend to zero as $N \rightarrow \infty$. In this respect the situation parallels that met in the proof of Ward identities.

An example will suffice to illustrate the argument. The simplest graph on the RHS of (5.3) containing a subgraph with 3 legs is shown in Fig. 4. 
The first few graphs on the RHS of (5.4c) are

Iterating once we get
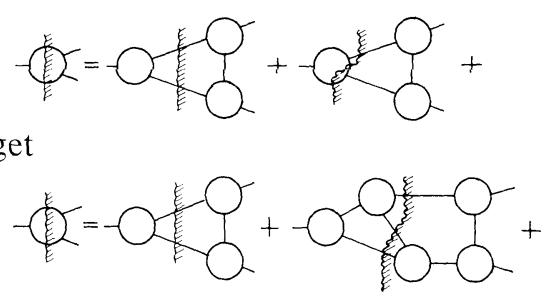

We see how the graph of Fig. 4 has emerged through the iteration.

With unitarity of the 3-point function established, we may now turn to the higher $n$-point functions, $n \geqq 4$. Consider a process $i \rightarrow f$, with all ingoing and outgoing "particles" having positive energy.

Applying Veltman's general cutting formula with only $y$-type arguments to the skeleton graph expansion one obtains a formula analogous to $(5.4 \mathrm{c})$ for the absorptive part of the amplitude. For the cut vertices in the term in \{\} (also those inside the boxes) one substitutes the already established unitarity relation for the 3-point functions. In this way one recovers just the RHS of Eq. (5.3). The first term in $(5.4 \mathrm{c})$ will again give those contributions without 3 -point subgraph, and the second term all the rest.

Lastly let us turn to unitarity of the 2-point function. We have already shown in Section 4 that Eqs. (2.11) and (3.19) are equivalent to the internal charge resp. momentum form of the propagator bootstrap (1.2). It suffices then to show that both Eq. (2.11) as well as (3.19) predict the same absorptive part for the (charged resp. arbitrary) propagator as the unitarity relation does. Equivalence then follows from the Lehmann representation, which predicts the real part uniquely. The algebra is exactly the same in both cases; thus we may treat Eq. (2.11) and (3.19) simultaneously.

Unitarity of the 2-point function can be written as (disc ... $\equiv-2$ Re ...)

$$
\text { disc }-1=\sum_{n \geqslant 2}
$$

The box notation amounts here simply to waiving all one particle irreducibility requirements. Thus, by distributing $\times$ 's in the familiar way (of Section 4)

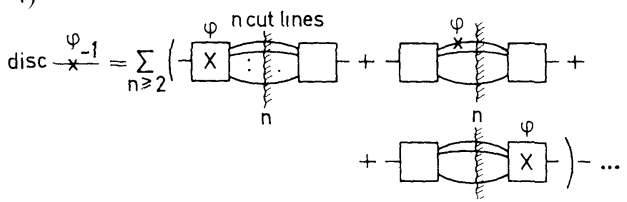

with $\times$ notation as explained in Eq. (1.2a). 
We now insert WTI (4.3) with $\varphi(x)=x^{v}$. This identity was proven in Section 4 from the vertex bootstrap and remains true when 1-particle irreducibility requirement is waived. One obtains

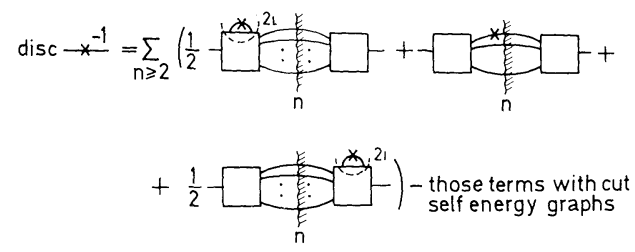

On the other hand, we obtain by applying Veltman's cutting formula to Eq. (2.11) resp. (3.19)

$$
\text { disc } *-1=\frac{1}{2}+\epsilon^{-1}+\frac{1}{2}
$$

If we substitute the 3-point unitarity relation for any cut vertex that may occur inside the ovals. we recover the RHS of (5.6). The 2-particle irreducibility property of the $B S$-kernel assures that there are no contributions with cut selfenergy subgraphs. This completes the proof of unitarity.

Finally, we will comment on the original renormalized propagator Eq. (4.22) of Ref. [16]

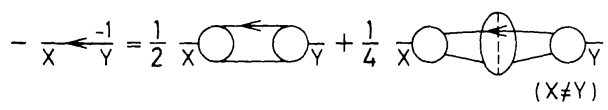

where the arrow is multiplication by $x_{\text {left }}-x_{\text {right }}$, the coordinate space form of momentum differentiation. In the last term on the r.h.s. we write, in view of the skeleton expansion of the BS-kernel,

$$
x_{\text {left }}-x_{\text {right }}=\left(x_{\text {left }}-y_{1}\right)+\left(y_{1}-y_{2}\right)+\cdots+\left(y_{n-1}-y_{n}\right)+\left(y_{n}-x_{1 \text { ight }}\right)
$$

where the differences multiply a vertex or a propagator. We now take the real part of both sides, using the Veltman's cutting formula. It is not difficult to see that what is obtained thereby is equivalent modulo vertex bootstrap to (5.5) multiplied by the external-coordinate difference. Thus, the propagator unitarity bootstrap is equivalent modulo vertex bootstrap to the bootstrap given by (5.8), and thus also equivalent to the bootstrap (2.11) and (3.19).

Acknowledgement. One of the authors (G. M.) wishes to thank the Deutsche Forschungsgemeinschaft for financial support, and Prof. H. Joos and DESY for hospitality 


\section{Appendix A}

\section{Explanation of Graphical Notation}

Basically, the notation is the one used by one of us in earlier papers $[15,16,27]$.

A round bubble with $n$ short legs attached to it represents an $n$-point vertex function $\Gamma(\ldots)$, i.e. the one-"particle" irreducible part of a connected, full-propagator-amputated Green function. An exception is the BS-kernel (Fig. 1.3) which may be one "particle" reducible in the crossed channels. A solid line represents a (dressed) propagator, a solid line marked with -1 an inverse propagator, which is the negative of the 2-point vertex function. Coordinates occuring as arguments of internal vertices are always understood to be integrated over. Crosses on lines or in bubbles signify the operations on those functions as are described in each case in the text.

One "particle" irreducibility means that no skeleton graphs are included which have one more disconnected component after one suitable line is cut. The concept can also be defined without reference to skeleton graphs, cf. Ref. [15].

The same is true, to a certain extent, of two- and three-"particle" irreducibility concepts which will be introduced presently.

A broken line with prescription $2 i$ and/or $3 i$ may be drawn through a function with $\geqq 4$ arguments (or, more generally, through a function that is defined by skeleton expansion). It means that those terms in the skeleton expansion are omitted which allow a two "particle" cut resp. three-"particle" cut that cuts only propagators and separates the arguments of the function into the indicated two groups. One specifies in addition, that a 3-"particle" cut in this sense may go through up to one displayed or external line.

We also use quadratic boxes. They stand for a sum of terms as follows ${ }^{13}$.

The vertex function as described before, plus all amputated and oneparticle reducible or even disconnected Green functions as far as they do not violate the overall connectedness and one-"particle"-irreducibility of the function being expressed in the equation the box is used in. A product of (inverse) propagators only is excluded however. Irreducibility prescriptions then refer to the entire possibly composite graph, i.e. three"particle" cuts to be excluded under $3 i$ - prescription may cut through lines belonging to different connected pieces inside a box or also through one external and/or one displayed line.

13 The box notation is used only to shorten the formulae. The reader may find it instructive to compare our graphical formulae with those of Ref. [25] where boxes in our notation are written out in terms of vertex functions. 
We give two examples: In Eq. (21.2) the quadratic box may be written out as follows

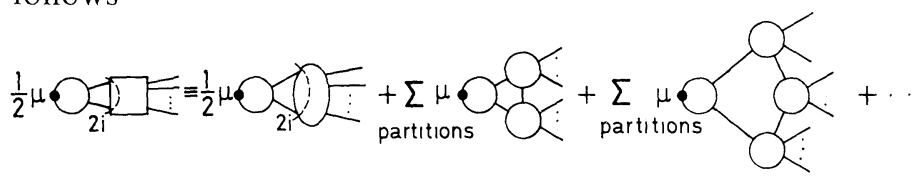

To illustrate the $3 i$-prescription let us note that in the last term of (4.4) contributions
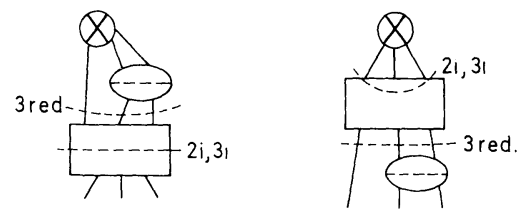

are not included. But

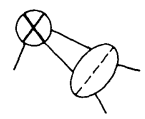

is included. It stems from a disconnected graph inside the box.

The proof of equations like (4.4) or (4.7) rests on the observation that the same graphs occur on both sides of the equation, and with the same multiplicity, such that also the symmetry numbers [23] are the same on both sides. The descriptions of the boxes in graphs with several of them, as in (4.5) and (4.8), follow from the box definition in the equations from which the former ones were obtained by insertion or iteration, in this case (4.3) and (4.4).

The notation and description of allowed contributions in the generalized unitarity graphs of Section 5 was given in the text.

\section{Appendix B}

Remark on Eq. (4.6)

In Section 4, the Ward Takahashi identity (4.6) for the 4-point vertex including a current was derived. From it one may conclude that

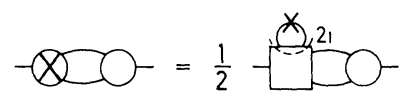

Now the LHS of (B.1) is infinite since it contains a subgraph that is divergent by the criteria of Ref. [5], irrespective of the value of dynamical dimension, as seen by applying the cross operation on Fig. 1 in Section 3. The individual skeleton graphs on the RHS of (B.1) are finite term by term, however. This is not a paradox; it only means that the series on the 
RHS will not converge (or, if one prefers, converges to infinity). This is possible because the terms on the RHS of (4.6) are not individually conformal covariant; they can, therefore, and will in fact, have a different $x$-dependence each. As a consequence, convergence (or, at least, meaningfulness) of the expansion on the RHS of (4.6) does not imply the same for the RHS of (B.1). In fact, inspection of the derivation of (B.1) reveals that, in obtaining the RHS, the neglected remainder term given in Fig. 5 is infinite.

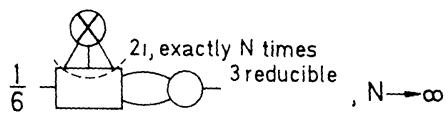

Fig. 5. A class of nonnegligible (since divergent) graphs

These remarks may have some practical importance, because our consideration implies that each of the invidual terms on the RHS of (4.6) have a lesser singularity as $x_{2}-x_{3} \rightarrow 0\left(x_{1}-x_{3}\right.$ fixed) than their sum, which is given by the LHS.

We add some fairly trivial remarks. In conformal field theory, unlike canonical perturbation theory, because of the bootstrap conditions one cannot make convergence assumptions wholly indiscriminately. To see this, recall that a series $\Sigma f_{N}(\ldots)$ can be convergent only if $f_{N}(\ldots) \rightarrow 0$ as $N \rightarrow \infty$. Consider now the vertex bootstrap (1.1). Iterating $N$ times gives the equivalent equation

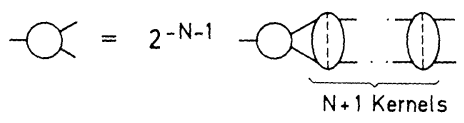

with $N$ arbitrary $\geqq 0$. Although the RHS involves only graphs of order $g^{n}, n \geqq 2 N+3$, it is manifestly false to assume that it tends to zero as $N \rightarrow \infty$. This fact, to be kept in mind, can be ascribed to the graphs on the RHS of (B.2) violating the skeleton condition. In this paper, no graphs violating the skeleton condition, except the defining bootstrap $\left(1.1^{\prime}\right)$ itself, occurred. In particular, a crossed vertex inside a three-point subgraph as in $\left(4.5^{\prime \prime}\right)$ must not be construed to violate the skeleton condition - even if the crossed vertex is proportional to the uncrossed one as would be the case for the cross operation signifying infinitesimal normalization change.

\section{Appendix C}

\section{Connection with the Finite-Mass Theory}

The conformal invariant theory allows to make statements possibly relevant to the real world if it is the Gell-Mann-Low (GL) limit [7] of a realistic finite-mass theory. For perturbation theoretically renormalizable 
theories (or, more generally, for certain families of Lagrangian QFTs parametrizable by a finite set of parameters) the assumptions necessary for such a limit theory to exist and the ensuing precise relationship to the finite-mass theory were discussed in Ref. [8] at the example of the $\phi^{4}$ theory. We briefly review these matters here, for brevity for the same model, referring to Ref. [8] as SD. The considerations are extensible (and in part have been extended by one of us) to the $p s-p s$ theory and to the $\phi^{3}$ theory in six dimensions.

To the (two-parameter) theory described by the $\Gamma\left(p_{1} \ldots p_{2 n} ; m^{2}, g\right)$, which satisfy partial differential equations (SD I.12), an (effectively oneparameter) zero-mass "preasymptotic" theory described by functions $\Gamma_{a s}\left(p_{1} \ldots p_{2 n} ; m^{2}, g\right)$ is associated. The $\Gamma_{a s}$ are the asymptotic forms of the $\Gamma$ if the momenta are nonexceptional (Section II of SD) and otherwise UR singular. They satisfy the homogeneous equations to (SD I.12) and approximate (for nonexceptional moments) the $\Gamma$ at large momenta.

This parametrization of the $\Gamma_{a s}$, though natural for asymptotic considerations, is inconvenient for e.g. their direct construction as zeromass-theory functions. One has (SD B.2)

$$
\Gamma_{a s}\left(p_{1} \ldots p_{2 n} ; m^{2}, g\right)=z(g)^{-n} \Gamma_{0}\left(p_{1} \ldots p_{2 n} ; m^{2}, V(g)\right)
$$

with a construction prescription for the $\Gamma_{0}$ in the spirit of Ref. [7], given in Appendix B of SD, in the notation of which the relation between $g$ and $V(g)$ is obtained by integrating $V^{2} R(V) d g=\beta(g) d V$, taking an integration constant there to from the $O\left(g^{2}\right)$ graphs.

The asymptotic form of the difference between $\Gamma$ and $\Gamma_{a s}$ is obtained by integrating (SD I.12) upon substituting for the inhomogeneous term its asymptotic form. The result is:

$$
\begin{aligned}
\Gamma\left(\lambda p_{1} \ldots\right. & \left.\lambda p_{2 n} ; m^{2}, g\right)-\Gamma_{a s}\left(\lambda p_{1} \ldots \lambda p_{2 n} ; m^{2}, g\right) \\
= & -i \lambda^{2-2 n} m^{2} a(g)^{n} h(g(\lambda)) a(g(\lambda))^{-n+1} e^{-\varrho(g)} \int_{0}^{g} d g^{\prime} \\
& \cdot \beta\left(g^{\prime}\right)^{-1} e^{\varrho\left(g^{\prime}\right)} a\left(g^{\prime}\right)^{-1} h\left(g^{\prime}\right)^{-1} \phi\left(g^{\prime}\right) \hat{\Gamma}_{\underline{a s}}\left(p_{1} \ldots p_{2 n} ; m^{2}, g(\lambda)\right) \\
& \left.+\lambda^{2-2 n} m^{2} a(g)^{n} h(g(\lambda))^{-1} a(g(\lambda))^{-n-1} e^{-\varrho(g)} \int_{0}^{g} d g^{\prime} \beta\left(g^{\prime}\right)^{-1} \quad \text { (C. } 2\right) \\
& \cdot e^{\varrho\left(g^{\prime}\right)}\left[k\left(g^{\prime}\right)-k(g(\lambda))\right] a\left(g^{\prime}\right)^{-1} h\left(g^{\prime}\right)^{-1} \phi\left(g^{\prime}\right) \Gamma_{\underline{a s}}\left(00 p_{1} \ldots p_{2 n} ; m^{2}, g(\lambda)\right) \\
& + \text { smaller terms as } \lambda \rightarrow \infty
\end{aligned}
$$

whereof the $n=1$ form is (SD III.27) with (SD III.7) and (SD IV.9) substituted on the RHS. The $\Gamma_{\underline{a s}}$ and $\hat{\Gamma}_{\underline{a s}}$ functions are defined in an 
analogous manner as the ones for $n=1$ in Section III and Appendix B of $\mathrm{SD}$. Next one requires the asymptotic $(\lambda \rightarrow \infty)$ behaviour of the $\Gamma_{a s}$ (and $\Gamma_{\underline{a s}}$ etc.) themselves, whereby

$$
\begin{aligned}
\Gamma_{a s}\left(\lambda p_{1} \ldots \lambda p_{2 n} ; m^{2}, g\right)= & \lambda^{4-2 n} \exp \left[-2 n \int_{g}^{g(\lambda)} d g^{\prime} \beta\left(g^{\prime}\right)^{-1} \gamma\left(g^{\prime}\right)\right] \\
& \cdot \Gamma_{a s}\left(p_{1} \ldots p_{2 n} ; m^{2}, g(\lambda)\right) .
\end{aligned}
$$

This is till the present only possible on the basis of assumptions. One assumes that $\beta(g)$ has a (first, but this is inessential) zero at $g_{\infty}$ for $g>0$. Then $\lim _{\lambda \rightarrow \infty} g(\lambda)=g(\infty) \equiv g_{\infty}$. One further assumes that (for nonexeptional momenta)

$$
\lim _{g \rightarrow g_{\infty}} \Gamma_{a s}\left(p_{1} \ldots p_{2 n} ; m^{2}, g\right) \equiv \Gamma_{\mathrm{GL}}\left(p_{1} \ldots p_{2 n} ; m^{2}\right)
$$

exists, and likewise the corresponding limit for the $\Gamma_{\underline{a s}}$ and $\hat{\Gamma}_{\underline{a} \mathbf{s}}$ in (C.2). The consistency of these assumptions is discussed in Section IV and Appendix B of SD. In the $\Gamma_{0}$-parametrization in (C.1), $g_{\infty} \leftrightarrow V\left(g_{\infty}\right) \equiv V_{\infty}$ is the bare coupling constant in a particular convention (in view of the nontrivial momenta dependence of the four-point $\Gamma_{\mathrm{GL}}$ ). The etc. have definite scaling properties, in particular,

$$
\Gamma_{\mathrm{GL}}\left(\lambda p_{1} \ldots \lambda p_{2 n} ; m^{2}\right)=\lambda^{4-2 n d} \Gamma_{\mathrm{GL}}\left(p_{1} \ldots p_{2 n} ; m^{2}\right)
$$

with $^{14} d=1+2 \lambda\left(g_{\infty}\right)=1+2 V_{\infty}^{2} R\left(V_{\infty}\right)>1$.

Assuming that $\beta(g), \gamma(g)$ and also the $\Gamma_{a s}\left(\ldots ; m^{2}, g\right)$ are continuously differentiable at $g \rightarrow g_{\infty}$ from below, one obtains by an elementary calculation (similar to the one leading to (SD IV.10)) from (C.3) with (C.4) and from (C.2), respectively, the following two correction terms for the asymptotic difference between $\Gamma$ and $\Gamma_{\mathrm{GL}}$ at nonexceptional momenta:

$$
\begin{aligned}
& \Gamma\left(\lambda p_{1} \ldots \lambda p_{2 n} ; m^{2}, g\right)-\lambda^{4-2 n d} r(g)^{-2 n} \Gamma_{\mathrm{GL}}\left(p_{1} \ldots p_{2 n} ; m^{2}\right) \\
& =-\left.\lambda^{4-2 n d+2 \beta^{\prime}\left(g_{\infty}\right)} c(g)\left[-2 n \beta^{\prime}\left(g_{\infty}\right)^{-1} \gamma^{\prime}\left(g_{\infty}\right)+\frac{\partial}{\partial g}\right] \Gamma_{a s}\left(p_{1} \ldots p_{2 n} ; m^{2}, g\right)\right|_{g=g_{\infty}} \\
& \quad+s(g)(\ln \lambda)^{\alpha} \lambda^{2-2 n d+2\left|\eta\left(g_{\infty}\right)+2 \gamma\left(g_{\infty}\right)\right|} f_{\mathrm{GL}}\left(p_{1} \ldots p_{2 n} ; m^{2}\right)+\cdots
\end{aligned}
$$

where $f_{\mathrm{GL}}$ is expressible in terms of the GL limit functions $\hat{\Gamma}_{\underline{a s}}\left(\ldots ; m^{2}, g_{\infty}\right)$ (cp. Appendix B of $S D$ ), $r(g), s(g)$ and $c(g)$ are $g$-dependent constants, and the dots stand for terms smaller at $\lambda \rightarrow \infty$ than the larger of the two

${ }^{14}$ Actually, one cannot exclude that $d=1$ and thus the GL theory being a free one [35]. We will disregard this possibility in the following. 
terms. Finally, $\alpha=1$ if $\eta\left(g_{\infty}\right)+2 \gamma\left(g_{\infty}\right)=0$ and $\alpha=0$ otherwise. From their defining formulas, one shows that

and

$$
\begin{gathered}
{\left.\left[\frac{\partial}{\partial g}-\cdots\right] \Gamma_{a s}\left(\lambda p_{1} \ldots \lambda p_{2 n} ; m^{2}, g\right)\right|_{g=g_{\infty}}} \\
=\left.\lambda^{4-2 n d+2 \beta^{\prime}\left(g_{\infty}\right)}\left[\frac{\partial}{\partial g}-\cdots\right] \Gamma_{a s}\left(p_{1} \ldots p_{2 n} ; m^{2}, g\right)\right|_{g=g_{\infty}}
\end{gathered}
$$

$f_{\mathrm{GL}}\left(\lambda p_{1} \ldots \lambda p_{2 n} ; m^{2}\right)=\lambda^{2-2 n d+2\left|\eta\left(g_{\infty}\right)+2 \gamma\left(g_{\infty}\right)\right|} f_{\mathrm{GL}}\left(p_{1} \ldots p_{2 n} ; m^{2}\right)$

which is consistent with (C.6) whichever is the larger correction term. (C.6) shows in which sense, and to what accuracy, the GL (Migdal) theory is the "asymptote" of the massive theory, and also of the preasymptotic $\Gamma_{a s}$ theory for which the second correction term in (C.6) would be absent. Thus, the vertex functions of all three theories have the same asymptotic behaviour.

All of this discussion can be taken over to the $p s-p s$ theory, and, in a certain formal way, to the $\phi^{3}$ theory in $D$ dimensions as treated in this paper, with the conclusion that also in these theories there are two asymptotic correction terms, one, $\Delta \Gamma_{1}=\left(\Gamma_{a s}-\Gamma_{\mathrm{GL}}\right)_{\mathrm{GL}}$ analogous to the first and one, $\Delta \Gamma_{2}=\left(\Gamma-\Gamma_{a s}\right)_{\mathrm{GL}}$ analogous to the second term on the RHS of (C.6), scaling with a lower power of the momenta than the $\Gamma_{\mathrm{GL}}$ themselves.

Now in these theories, described by $\Gamma, \Gamma_{\text {as }}$ and $\Gamma_{\mathrm{GL}}$, the fundamental dynamical equations, such as the vertex bootstrap (1.1) and generalized unitarity of the two-point function (5.5), are exactly the same in all three theories. It is only the additional requirement of conformal invariance (which plays the role of boundary condition) that singles out the particular solution studied in the body of this paper, while the UV convergence arguments of Ref. [5] apply to all three solutions. E.G., the RHS of (1.1) will be overall convergent in momentum space, and thus a bare vertex in (1.1) will be absent in all cases since it would violate the analog of (C.6) with $d>d_{\text {canonical }}$.

Furthermore, all algebraic manipulations of Sections 4 and 5 and their results (equivalence relations, Ward-like identities (4.6) etc.) apply to all three theories, to the extent that conformal invariance or the tracelessness of the stress tensor are not explicitly used.

This suggests to define two cross operations: replacing a vertex function of the conformal invariant theory by either $\Delta_{1} \Gamma$ or by $\Delta_{2} \Gamma$ as defined before, and replacing a propagator, the negative inverse of the two-point vertex function, correspondingly. We now start from the two-point unitarity Eq. (5.5) for the finite-mass or for the preasymptotic theory and 
go through the steps of Sections 4 and 5 to recover ${ }^{15}$ (2.11), where the BS kernel, and the uncrossed propagator in the relation between -- $x$ and $-x^{-1}$, are the conformal invariant Migdal ones ${ }^{16}$. The UV convergence criteria of Ref. [5] and a simple UR estimate in configuration space yield that for noncoinciding arguments the RHS of (2.11) is finite, if for the negative inverse propagator in $\phi^{3}$ theory in $D$ dimensions,

i.e.

$$
\Delta_{1.2} \Gamma(\lambda \times 0)=\lambda^{-2 D+2 d+2 \varepsilon_{1,2}} \Delta_{1.2} \Gamma(x 0)
$$

$$
\Delta_{1.2} G(\lambda x 0)=\lambda^{-2 d+2 \varepsilon_{12}} \Delta_{1.2} G(x 0)
$$

with $0<\varepsilon<\frac{1}{2} D$.

The homogeneous dilatation covariant integral Eq. (2.11) has a nontrivial solution only if $\varepsilon$ is a root of an algebraic equation similar to those determining dynamical dimensions of the fields. Thus, two (not necessarily distinct) $\operatorname{roots}^{17} \varepsilon_{1.2}$ determine the powers of the correction terms to the propagators, and from these, the $\Delta_{1.2} \Gamma$ for all other vertex functions can be computed from (4.6), with the box on the RHS provided by the conformal invariant theory. The correction terms will come out dilatation covariant, conforming with (C.7), but not conformal invariant.

For $\phi^{3}$-theory, the scaling behavior of the mass correction term $\Delta_{2} \Gamma(\mathrm{x} 0)$ to the inverse propagator can be determined explicitly. The result is $\varepsilon_{2}=\frac{1}{2} d$. To see this we note that we can immediately write down two solutions of Eq. (2.11) understood as an equation for $\Delta \Gamma(p,-p)$. They have $\varepsilon=\frac{1}{2}(D-d)$ and $\varepsilon=\frac{1}{2} d$ respectively. The second of these corresponds to $\varepsilon_{2}$ as defined above. These solutions are provided by the validity of the vertex bootstrap condition (1.1) as is seen by letting in (1.1) the left argument go to infinity appropriately or, alternatively, integrating over that argument.

The point of this Appendix is that, in principle, starting from the conformal invariant theory, one can calculate quantities transcending this theory.

Note Added in Proof. (1) In Migdal's formula for the 3-point function of a current and two Dirac fields, Eq. (2.8 b), only two of the three terms in the sum are actually independent. One should therefore put $a_{T}=0$ in Eq. $(2.8 \mathrm{~b})$ and the following discussion. The authors are grateful to Drs. Dobrev, Stamenov and I. Todorov for this remark.

The correct expression for the 3-point function involving arbitrary currents and tensor fields was exhibited by Nobili [Padova preprint IFPTH-1/72] and Ferrara, Gatto, Grillo

15 As usual. there is a convergence assumption involved here: Eq. (4.4), with crossed propagators and vertices as specified above, must allow iterative solution for the crossed vertex. Such assumption cannot, if the GL theory is involved, be verified by perturbation theory, and also the arguments given after $\left(4.5^{\prime \prime}\right)$ do not now apply.

${ }^{16}(2.11)$ is analogous to equations used by Baker and Johnson [36] in QED, cp. also Ref. [37]. - (2.11) would also hold in $\phi^{4}$ theory, for which (C.6), (C.7) were derived.

17 A double root would allow a single $\ln \lambda$ factor as appears in (C.6) for $\alpha=1$.

19 Commun math Phys, Vol 27 
and Parssi [in: Springes Tracts, to appear]. Their result contains in particular also our Eq. (3.8) for the stress tensors 3-point Wightman function, and the remark of footnote 8.

This last result also fits in with a general theorem of Ferrara, Gatto, Grillo and Pariss [Phys. Letters $38 \mathrm{~B}, 333(1972)$ ] It says that a traceless s-rank tensor ficld $B_{\mu_{1}}, \mu_{s}(x)$ with canonical dimension $d=D+s-2$ is automatically conserved, assuming that its 2-point function $\left\langle 0\left|B_{\mu_{1}} \mu_{s}(x) B_{v_{1}} \ldots v_{s}(0)\right| 0\right\rangle$ is conformal invariant. (In the present approach, these 2-point functions would have to be computed from Eys. analogous to (2.15) which are not manifestly conformal invariant because of the crosses, though.)

(2) We have occasion to emphasize that the argument given after Eq. (4.5) for the nonexistence of a solution of the homogeneous equation associated with (4.4) depends on charge $Q$ being conserved at every vertex. Expression (4.4) then involves only differences $\varphi\left(x_{1}\right)-\varphi\left(x_{j}\right)$ which tend to zero when $x_{i} \rightarrow x_{j}$. This makes the subintegrations in (4.4) sufficiently well convergent for the stated holomorphy property of its RHS in $d$ to hold.

(3) In the equation preceding (49), subscript $e$ on $D_{e}$ and in the sum should read 1 . (Throughout the paper $D_{1}$ means the operation of attaching a cross to line 1 , while $D_{t}$ attaches a cross to vertex $v$. Summations are as explained after Eq. (1.4).)

\section{References}

1. Migdal, A. A.: Phys. Letters 37 B, 98 (1971).

2. - Phys. Letters 37 B, 386 (1971).

3. Polyakov, A. M.: Zh ETF Pis. Red. 12. 538 (1970); - Transl. JETP Letters 12, 381 (1970).

4. Parisi, G., Peliti.L.: Lettere Nuovo Cimento 2, 627 (1971).

5. Mack, G., Todorov, I. T.: IC/71/139, Trieste, Oct. 1971.

6. Schroer, B.: Lettere Nuovo Cimento 2, 867 (1971).

7. Gell-Mann, M., Low, F.E.: Phys. Rev. 95, 1300 (1954). -- Wilson, K. G.: Phys. Rev. D 3, 1818 (1971).

8. Symanzik, K.: Commun. math. Phys. 23, 49 (1971).

9. Wess, J.E.: Nuovo Cimento 18, 1086 (1960). - Kastrup, H. A.: Ann. Physik 7, 388 (1962) and references cited in [11].

10. Mack, G.: Nucl. Phys. B 5, 499 (1968).

11. - Salam, A.: Ann. Phys. (N. Y.) 53, 174 (1969).

12. Wilson, K. G.: Phys. Rev. 179, 1499 (1969).

13. Schroer, B.: Fortschr. Physik 11, 1 (1963).

14. Glaser, V., Lehmann, H., Zimmermann. W.: Nuovo Cimento 6, 1122 (1957).

15. Symanzik, K.: J. Math. Phys. 1, 249 (1960). In: Lectures in Theoretical physics, Vol. III, ed. W.E. Brittin et al., p. 490. New York: Interscience Publ. 1961. In: Ramakrishnan, A., (Ed.): Symposia on Theoretical Physics, Vol. 3, p. 121. New York: Plenum Press 1967.

16. - In: Jaśič, (Ed.): Lectures on High Energy Physics. Zagreb: 1961, New York: Gordon and Breach 1965.

17. Schwinger, J.: Proc. Natl. Acad. Sci. US 44, 956 (1958). - Symanzik, K.: J. Math. Phys. 7, 510 (1966).

18. Hortacsu, M., Seiler, R., Schroer, B.: NYO-3829-80, U. of Pittsburgh, Sept. 1971.

19. Brandt, R. A.: Ann. Phys. 52, 122 (1969).

20. Symanzik, K.: Lettere Nuovo Cimento 3, 734 (1972).

21. Epstein,H., Glaser,V.: CERN Th. 1156, Geneva, May 1970. -- Steinmann, O.: Perturbation expansions in axiomatic field theory. Lecture Notes in Physics, Vol. 11. Berlin-Heidelberg-New York: Sprınger 1971. 
22. Polyakov, A. M.: Zh ETF 59, 542 (1970); - Transl. JETP 32, 296 (1971).

23. Wu,T.T.: Phys. Rev. 125, 1436 (1962).

24. Taylor, J. G.: Suppl. al Nuovo Cimento 1,857 (1963).

25. Johnson, R. W.: J. Math. Phys. 11, 2161 (1970).

26. Symanzik, K.: Commun. math. Phys. 6, 228 (1967).

27. - In: Bessis,J.D., (Ed.): Cargèse Lectures in Physics, Vol. 6. New York: Gordon and Breach 1971.

28. D’Eramo, M., Peliti, L., Parisi, G.: Lettere Nuovo Cimento 2, 878 (1971).

29. Gatto, R.: Riv. Nuovo Cimento 1, 514 (1969).

30. Callan, C. G., Jr., Coleman, S., Jackiw, R.: Ann. Phys. 59, 42 (1970).

31. Speer,E.R.: Generalized Feynman amplitudes. Princeton: Princeton University Press 1969.

32. Mack, G.: Expansions around canonical dimensions in conformal invariant Quantum field theory II (in preparation).

33. Bogolubov, N.N., Shirkov,D.V.: Introduction to the theory of quantized fields. $\$ 17.5$. New York: Intersc. Publ. 1959.

34. Veltman, M.: Physica 29, 186 (1963).

35. Pohlmeyer, K.: Commun. math. Phys. 12, 204 (1969).

36. Baker, M., Johnson, K.: Phys. Rev. 183, 1292 (1969); D 3, 2516 (1971).

37. Adler, S. L., Bardeen, W. A.: Phys. Rev. D 4, 3045 (1971).

K. Symanzik

DESY

Notkestieg 1

D-2000 Hamburg 52

Federal Republic of Germany
G. Mack

Universität Bern

Institut für Theoretische Physik

Sidlerstr. 5

$\mathrm{CH}-3000$ Bern Switzerland 
